Cita: Morquecho-Sánchez, R.; Morales-Sánchez, V.; Ceballos, O.; Pineda-Espejel, H.A.; Zamarripa, J. (2021). Evaluación de la calidad percibida de los programas de voluntariado deportivo universitario al contexto mexicano: Universiada Nacional. Cuadernos de Psicología del Deporte, 21(2), 174-182

\title{
Evaluación de la calidad percibida de los programas de voluntariado deportivo universitario al contexto mexicano: Universiada Nacional
}

\section{Evaluation of the perceived quality of university sports volunteering programs in the Mexican context: National Universiade.}

\section{Avaliação da percepção da qualidade dos programas de voluntariado desportivo universitário no contexto mexicano: Universiade Nacional}

\author{
Morquecho-Sánchez, R. ${ }^{1}$, Morales-Sánchez, V. ${ }^{2}$, Ceballos, O. ${ }^{1}$, Pineda-Espejel, H.A. ${ }^{3}$, Zamarripa, J. ${ }^{1}$ \\ ${ }^{1}$ Universidad Autónoma de Nuevo León, Facultad de Organización Deportiva; ${ }^{2}$ Universidad de Malaga, \\ Facultad de Psicología; ${ }^{3}$ Universidad Autónoma de Baja California, Facultad de Deportes.
}

\begin{abstract}
RESUMEN
El objetivo de la presente investigación es validar una herramienta que permita evaluar la calidad percibida de los programas del voluntariado universitario al contexto deportivo mexicano. Para el análisis de los datos se utilizó el software estadístico SPSS v.22 y el programa LISREL 8.8. Se realizaron los análisis de consistencia interna, análisis factorial exploratorio (AFE) y análisis factorial confirmatorio (AFC) con dos muestras distintas. El instrumento utilizado fue el QVOLSPORT mx, el cual está compuesto por 49 ítems y 6 factores: 1. Organización del voluntariado, 2. Responsables de área, 3. Tareas específicas del área, 4. Logística, 5. Instalación y materiales y 6.Valoración personal. El muestreo fue intencional por conveniencia, los participantes son voluntarios deportivos universitarios de la Universiada Nacional del año 2017 y 2019 de ambos géneros. Se aplicó a una muestra de 778 participantes distribuida en 378 participantes en la Universiada del año 2017, donde 252 fueron hombres y 126 mujeres, mientras que para la Universiada del año 2019 participaron 400, donde 238 son hombres y 162 mujeres, con un rango de edad entre 18 y 31 años $(M=21.18)$. Los resultados mostraron que el Alfa de Cronbach por factores está por encima de .70; los indicadores de pertinencia fueron adecuados, los 6 factores obtenidos explican el 59.60\% de la varianza total. Los índices de ajuste y de error son satisfactorios, $\mathrm{CFI}=0.982, \mathrm{NNFI}=0.981$ y RMSEA $=0.057$, por lo que el modelo ajusta. Se concluye que el instrumento QVOLSPORT mx, presenta propiedades psicométricas adecuadas y atiende a las necesidades del voluntariado deportivo de la comunidad universitaria, posibilitando una mejora continua en los programas de voluntariado deportivo. Se confirma que el instrumento es válido para evaluar la calidad percibida en los programas del voluntariado deportivo universitario al contexto deportivo mexicano.
\end{abstract}

Palabras clave: Validación, Calidad percibida, Voluntarios, Evento deportivo, Universidad.

\section{ABSTRACT}

The objective of this research is to validate a tool that allows evaluating the perceived quality of university volunteer programs in the Mexican sports context. For data analysis, the statistical software SPSS v.22 and the LISREL 8.8 program were used. The internal consistency analyzes, exploratory factor analysis (AFE) and confirmatory factor 


\section{Morquecho et al.}

analysis (AFC) were performed with two different samples. The instrument used was the QVOLSPORT mx, which is composed of 49 items and 6 factors: 1 . Organization of the volunteer work, 2. Person in charge of the area, 3. Specific tasks of the area, 4. Logistics, 5. Installation and materials and 6. Personal assessment. The sampling was intentional for convenience, the participants are university sports volunteers of the National Universiade of the year 2017 and 2019 of both genders. It was applied to a sample of 778 participants distributed in 378 participants in the 2017 Universiade, where 252 were men and 126 women, while for the 2019 Universiade 400 participated, where 238 were men and 162 women, with a range of age between 18 and 31 years $(M=21.18)$. The results showed that Cronbach's Alpha by factors is above .70; the indicators of relevance were adequate, the 6 factors obtained explain $59.60 \%$ of the total variance. The fit and error indices are satisfactory, CFI $=0.982$, NNFI $=0.981$ and RMSEA $=$ 0.057 , so the model fits. It is concluded that the QVOLSPORT $\mathrm{mx}$ instrument has adequate psychometric properties and meets the needs of sports volunteering in the university community, enabling continuous improvement in sports volunteering programs. It is confirmed that the instrument is valid to evaluate the perceived quality of university sports volunteering programs in the Mexican sports context.

Keywords: Validation, Perceived quality, Volunteers, Sports event, University.

\section{RESUMO}

O objetivo desta pesquisa é validar uma ferramenta que permite avaliar a qualidade percebida dos programas de voluntariado universitário no contexto esportivo mexicano. Para a análise dos dados, foram utilizados os softwares estatísticos SPSS v.22 e LISREL 8.8. As análises de consistência interna, análise fatorial exploratória (AFE) e análise fatorial confirmatória (AFC) foram realizadas com duas amostras diferentes. O instrumento utilizado foi o QVOLSPORT mx, que é composto por 49 itens e 6 fatores: 1. Organização do trabalho voluntário, 2. Responsável pela área, 3. Tarefas específicas da área, 4. Logística, 5. Instalação e materiais e 6. Avaliação pessoal. A amostragem foi intencional por conveniência, os participantes são voluntários do esporte universitário da Universiade Nacional dos anos de 2017 e 2019 de ambos os sexos. Foi aplicado a uma amostra de 778 participantes distribuídos em 378 participantes na Universiade 2017, sendo 252 homens e 126 mulheres, enquanto na Universiade 2019 participaram 400, sendo 238 homens e 162 mulheres, com faixa etária entre 18 e 31 anos $(M=21,18)$. Os resultados mostraram que o Alfa de Cronbach por fatores está acima de 0,70; os indicadores de relevância foram adequados, os 6 fatores obtidos explicam 59,60\% da variância total. Os índices de ajuste e erro são satisfatórios, $\mathrm{CFI}=0,982, \mathrm{NNFI}=0,981$ e RMSEA $=0,057$, então o modelo se ajusta. Concluise que o instrumento QVOLSPORT mx apresenta propriedades psicométricas adequadas e atende às necessidades do voluntariado esportivo na comunidade universitária, possibilitando a melhoria contínua nos programas de voluntariado esportivo. Confirmase que o instrumento é válido para avaliar a qualidade percebida de programas de voluntariado esportivo universitário no contexto esportivo mexicano.

Palavras-chave: Validação, Qualidade percebida, Voluntários, Evento esportivo, Universidade.

\section{INTRODUCCION}

La imagen del voluntario en la sociedad posee una función que en los último años ha cobrado interés para su estudio desde las Ciencias Sociales (González et al, 2011; Littman Ovadia y Steger, 2010). Diversos autores, muestran cómo esta figura se vuelve relevante al realizar eventos deportivos e incluso para implementar una política pública (García Pérez, 2013; Vecina et al, 2009; Miranda y Mayne Nicholls, 2009;
Nicholls et al, 2014). De esta forma, el voluntariado es una función social importante en diferentes campos sociales, como el deporte, por ejemplo, es un contexto en donde la participación de los voluntarios representa un rol importante para las actividades que desarrollarán con su fuerza de trabajo.

Según Chica Merino (2009), el voluntariado se define como una decisión que se toma de forma reflexiva, responsable, por iniciativa propia, supone un compromiso y se ejerce de forma desinteresada. 


\section{Calidad en programas de voluntariado de la Universiada mexicana}

De acuerdo con Morales Sánchez et al (2020), para mejorar la experiencia emocional de los usuarios en las organizaciones, se deben considerar varias cuestiones. Por ejemplo, la interacción entre trabajadores y usuarios será de vital importancia, y debe fomentarse una buena comunicación entre ellos si se quiere incrementar la experiencia de emociones positivas, esto es lo que también se busca lograr entre el voluntariado deportivo y las organizaciones deportivas.

El voluntariado es una actividad que ha incrementado su importancia en la última década en la promoción y organización de eventos deportivos (Brotóns y Molina, 2011). Uno de los países que cuenta con una mayor proporción de voluntariado es el Reino Unido, resaltando la necesidad de la participación ciudadana para beneficiar tanto a la sociedad individual como a la sociedad en general con su idea política "Big Society", con una participación de 64,000 clubes deportivos administrados por voluntarios en los que casi 1,5 millones de voluntarios apoyar a más de 5.3 millones de jóvenes participantes en Inglaterra (Nichols, Taylor, Barrett, y Jeanes, 2014); sin embargo, se enfrentan a diferentes retos que les dificultan su participación por la falta de apoyo del mismo gobierno y por recortes en el gasto público (Morgan, 2013; Taylor, Panagouleas, y Nichols, 2012).

En este contexto y con un mayor alcance, se crean las estrategias de voluntariado para los juegos olímpicos y paralímpicos de Tokio 2021, centradas en el reclutamiento y entrenamiento, iniciando desde verano del 2018, donde se espera que los interesados especialmente estudiantes se involucren para colaborar en diferentes actividades; los voluntarios que estarán participando en la organización serán identificados como "Games Volunteers", un segundo grupo "City Volunteers" estará colaborando en aeropuertos, estaciones de tren, señalizaciones y localizaciones para brindar servicios a los visitantes. Como referencia del alcance de este tipo de eventos, se tiene la experiencia de los Juegos de Londres 2012 donde aplicaron para el primer grupo "Games Makers" 240,000 y fueron aceptados 70,000 y en el segundo "London Ambassadors" aplicaron 24,000 y quedaron 8,000, contribuyendo satisfactoriamente al éxito del magno evento.
La gran demanda de eventos deportivos provoca una mayor importancia de la gestión dentro de las organizaciones de dichos eventos y la necesaria elaboración de planes estratégicos de gestión para buscar la calidad, el bienestar de estos eventos y actividades deportivas (García-González, MoralesSánchez, Hernández-Mendo y Chica-Merino, 2011). Dentro de dichos planes es de vital importancia considerar para el éxito de los mismos un programa de voluntariado.

Por otro lado, el evento deportivo universitario internacional es organizado cada dos años por la Federación Internacional del Deporte Universitario (FISU), donde estudiantes de todo el mundo tienen la posibilidad de adquirir experiencia en un evento deportivo internacional y desarrollar habilidades de comunicación y gestión de eventos, al participar en el programa de voluntariado (FISU, 2015).

La Universiada Nacional, el evento deportivo en la cual se centra la presente investigación, es la justa deportiva estudiantil más importante en México que se realiza de forma bianual, ya que reúne a los mejores atletas de instituciones de educación superior públicas y privadas. Participar en ella significa el inicio de una carrera deportiva en el alto rendimiento; ya que representa la posibilidad de competir en la Universiada Mundial, en los Juegos Centroamericanos y del Caribe, los Juegos Panamericanos, así como a nivel olímpico. En estos torneos, el buen desarrollo de la gestión de los eventos que involucran una gran cantidad de disciplinas deportivas, requiere de una planeación, ejecución y en especial, de la evaluación de la calidad de la organización donde se ve involucrado el voluntariado como un soporte estratégico.

Según Gallardo et al (2016), en los últimos años, una de las estrategias para fomentar este tipo de actividades han sido los programas o eventos deportivos basados en recomendaciones y buenas prácticas en materia de salud. Los gastos de la mayor parte de estos eventos o programas deportivos son asumidos por empresas e instituciones. Estos programas no sólo tienen una finalidad de competencia y saludable, sino que también realizan una gran labor social. Sin embargo, muchos de estos 


\section{Morquecho et al.}

programas no aplican ningún tipo de herramienta de evaluación sobre la calidad.

Este trabajo se basa en la investigación de los autores García-González, Chica-Merino, Hernández-Mendo y Morales-Sánchez (2011), los cuales señalan que la evaluación de la calidad percibida del voluntariado deportivo, es una tarea necesaria y de gran relevancia desde la responsabilidad que supone una buena organización, ya que permite identificar las áreas de oportunidad, mejorar la imagen del servicio, incrementar la atracción de nuevos participantes y aportar conocimiento hacia la gestión del deporte, particularmente en México y Latinoamérica. Por lo que se considera necesario un instrumento que evalúe específicamente la calidad percibida de los programas de voluntariado deportivo universitario al contexto mexicano.

El objetivo de ésta investigación, es validar una herramienta que permita evaluar la calidad percibida de los programas del voluntariado deportivo universitario al contexto mexicano.

\section{MATERIAL Y MÉTODOS}

El diseño de la investigación se trata de un diseño empírico, asociativo, evolutivo de carácter comparativo, (Anguera y Hernández-Mendo, 2003; Ato et al, 2013).

\section{Participantes}

La muestra fue intencional por conveniencia, en dos momentos diferentes y la misma justa deportiva, los participantes son voluntarios deportivos de la Universiada Nacional del año 2017 y 2019 de ambos géneros.

En la investigación se muestrearon a 378 participantes en la Universiada del año 2017, donde 252 fueron hombres y 126 mujeres, mientras que para la Universiada del año 2019 participaron 400, donde 238 son hombres y 162 mujeres, dando un total de ambas muestras de 778 participantes, con un rango de edad entre 18 y 35 años $(M=21.18)$.

\section{Medidas}

El instrumento utilizado fue el QVOLSPORT, el cual presenta una fiabilidad óptima, dado que los valores del alpha de Cronbach varían entre 0.72 para la escala
1 y 0.84 para la 2, diseñado por García-González, Chica-Merino, Hernández-Mendo y Morales-Sánchez (2011), el cuestionario está destinado a evaluar la calidad percibida de los programas de voluntariado deportivo, está compuesto por 49 ítems y seis factores: 1. Organización del voluntariado, 2. Responsables de área, 3. Tareas específicas del área, 4. Logística, 5. Instalación y materiales y 6 .Valoración personal. Las opciones de respuesta son tipo likert de 1 a 4 , donde 1 es nada de acuerdo y 4 muy de acuerdo.Measures of Swimming Performance

Swimming performance was assessed using the FINA points system which allows comparisons of performances regardless of technique or distance swam (FINA, 2011). To that end, the participant's coach provided information regarding each athlete's $100 \mathrm{~m}$ freestyle personal best swim time. Then, each time was converted to FINA points, a standard measure of international swimming performance where 1000 points represents the average of the top 10 all-time results in each event.

\section{Procedimiento}

Se ha utilizado el método Delphi y análisis metodológicos como la consistencia interna y análisis factoriales, para la validación del QVOLSPORT mx al contexto deportivo mexicano. Con respecto a los análisis metodológicos, la primera muestra se utilizó para realizar el análisis factorial exploratorio (AFE) y en la segunda muestra para el análisis factorial confirmatorio (AFC). Para la aplicación del método Delphi, se estableció un formato de respuesta para realizar la validación. Después de tres semanas se recopiló la retroalimentación de la validación de las comisiones de expertos y se realizaron las adaptaciones al instrumento conforme a la retroalimentación recibida.

Una vez validado el instrumento por los expertos, se comenzó con la solicitud de autorización a los miembros del Comité Organizador de la justa deportiva nacional, las credenciales y la capacitación de los encuestadores. Se tomó la decisión de aplicar el mismo instrumento en dos momentos diferentes en el año 2017 y 2019, pero en la misma justa deportiva.

Se aplicó el siguiente protocolo y criterios de inclusión: los participantes deben ser voluntarios deportivos universitarios activos dentro de las 


\section{Calidad en programas de voluntariado de la Universiada mexicana}

entidades deportivas universitarias donde se realizó la justa deportiva, respondían el cuestionario una vez que terminaban sus actividades dentro de la instalación deportiva de forma individualizada y voluntaria; siempre en presencia de la investigadora o los encuestadores. A los voluntarios se les explicaba que su participación sería anónima y desinteresada.

\section{Análisis de datos}

Se procesaron los datos por medio de los programas estadísticos SPSS versión 22 y el programa LISREL8.8. La fiabilidad del instrumento se calculó a través del coeficiente Alfa de Cronbach. Se estimó el coeficiente Kaiser-Meyer-Olkin (KMO) y la prueba de esfericidad de Bartlett, así como un análisis de matriz de correlaciones con el objetivo de determinar la pertinencia de dichos análisis (Visauta, 1998).

Se realizaron análisis factoriales, el cual es uno de los procedimientos estadísticos más utilizados en la investigación social, de los cuales se obtuvieron los índices de error y de ajuste.

\section{RESULTADOS}

Se realizó un análisis de componentes de varianza utilizando un procedimiento de máxima verosimilitud (ML) y de mínimos cuadrados (VARCOMP), el error residual es igual en ambos procedimientos, estos resultados permiten asumir que la muestra es lineal, normal y homocedástica (Hemmerle y Hartley, 1973; Searle, Casella, y McCulloch, 1992).

\section{Análisis de consistencia interna}

La consistencia interna fue calculada mediante el índice alfa de Cronbach, en lo que se refiere a los factores resultantes que componen el instrumento. El análisis de fiabilidad reveló una buena consistencia interna, mostrando valores en este caso superiores a 0.70 en cada factor (Cronbach, 1951), entre un rango de 0.79 y 0.92 (ver tabla 1 ).

El análisis de correlación mostró que todos los factores (los responsables del área, valoración personal, la organización del voluntariado, tareas específicas del área, instalaciones /materiales y logística) se correlacionan de manera positiva entre ellos y están significativamente relacionados $(\mathrm{p}<.01)$.

Se presenta la matriz de correlación de estructura por factores donde se muestra que todas las relaciones son significativas a $\mathrm{p}<.01 \mathrm{y}$ los factores discriminan entre ellos (ver tabla 2).

Tabla 1. Media, desviación típica, fiabilidad y correlaciones entre las variables

\begin{tabular}{lcccccccc}
\hline Factor & $M$ & $D T$ & 1 & 2 & 3 & 4 & 5 & 6 \\
\hline 1. Los/as responsables de área & 3.18 & .61 & $(.92)$ & & & & & \\
2. Valoración personal & 3.31 & .60 & $.494^{* *}$ & $(.89)$ & & & \\
3. La organización del voluntariado & 2.98 & .67 & $.559^{* *}$ & $.423^{* *}$ & $(.88)$ & & & \\
4. Tareas específicas del área & 3.02 & .65 & $.640^{* *}$ & $.518^{* *}$ & $.526^{* *}$ & $(.85)$ & \\
5. Instalaciones y materiales & 3.19 & .65 & $.589^{* *}$ & $.592^{* *}$ & $.463^{* *}$ & $.531^{* *}$ & $(.84)$ & \\
6. Logística & 3.06 & .68 & $.485^{* *}$ & $.535^{* *}$ & $.486^{* *}$ & $.565^{* *}$ & $559 . *$ & $(.79)$ \\
\hline
\end{tabular}

Nota: $\mathrm{M}=$ Media, DT = Desviación estándar, Alfa de Cronbach sobre la diagonal; ${ }^{* *} p<.01$. 


\section{Morquecho et al.}

Tabla 2. Matriz de correlación de estructura por factores QVOLSPORTmx.

\begin{tabular}{|c|c|c|c|c|c|c|}
\hline Factores & s OR & RA & TA & $\mathrm{LO}$ & IM & VP \\
\hline OR & 1.000 & & & & & \\
\hline RA & 0.642 & 1.000 & & & & \\
\hline$(0.031)$ & & & & & & \\
\hline 20.904 & & & & & & \\
\hline $\mathrm{TA}$ & 0.646 & 0.785 & 1.000 & & & \\
\hline$(0.034)$ & $(0.023)$ & & & & & \\
\hline 19.162 & 34.017 & & & & & \\
\hline LO & 0.565 & 0.580 & 0.720 & 1.000 & & \\
\hline$(0.035)$ & (0.039) & $(0.031)$ & & & & \\
\hline 16.135 & 15.006 & 23.393 & & & & \\
\hline & 0.604 & 0.673 & 0.729 & 0.758 & 1.000 & \\
\hline$(0.032)$ & $(0.034)$ & $(0.031)$ & $(0.027)$ & & & \\
\hline 18.842 & 19.976 & 23.496 & 27.942 & & & \\
\hline VP & 0.566 & 0.582 & 0.695 & 0.658 & 0.681 & 1.000 \\
\hline$(0.034)$ & $(0.037)$ & (0.037) & $(0.034)$ & $\quad(0.030$ & & \\
\hline 16.622 & 15.577 & 18.807 & 19.625 & 22.45 & & \\
\hline
\end{tabular}

\section{Estudio 1}

\section{Análisis factorial exploratorio (AFE)}

Para comprobar la adecuación del instrumento, se realizó un análisis factorial exploratorio con la muestra obtenida en el año 2017, sobre los 49 ítems; se comprobó la pertinencia de la muestra, por medio de la prueba de esfericidad de Bartlett, y el índice de adecuación de la muestra de Kaiser-Meyer-Olkin (KMO). El valor de la medida de adecuación muestral fue óptimo, con un índice de KMO de 0.93; y la prueba de Bartlett resultó estadísticamente significativa con un valor $\left(\mathrm{x}^{\wedge} 2=9365.7, \mathrm{gl}=861 ; \mathrm{p}<.001\right)$. Se extrajeron 6 factores los cuales conjuntamente, explican un $59.60 \%$ de la varianza total explicada (ver tabla 3).

Tabla 3. Criterios de pertenencia del Análisis Factorial Exploratorio (AFE)

\begin{tabular}{ll}
\hline Criterios de Pertinencia AFE & QVOLSPORT mx \\
\hline & \\
Determinante & .000 \\
KMO & 0.93 \\
SIG. & .000 \\
Prueba de esfericidad de Bartlett/chi-cuadrado & $\mathrm{x}^{2}=9365.7, g l=861 ; p<.001$ \\
Varianza Total Explicada & $59.60 \%$ \\
\hline
\end{tabular}

\section{Estudio 2}

Análisis factorial confirmatorio (AFC)

Con la muestra obtenida durante el año 2019, se realizó el AFC donde se presentan los resultados del modelo, con el objetivo de comprobar la estructura factorial. Con respecto a los índices de ajuste se encuentran por encima de .90 , lo que indica que es modelo es bueno y se ajusta a la realidad. Lo cual representa que el ajuste global del modelo es adecuado (ver tabla 4). 


\section{Calidad en programas de voluntariado de la Universiada mexicana}

Tabla 4.

Índices de Ajuste y Error de QVOLSPORT mx

\begin{tabular}{ll}
\hline Índices de ajuste y error & QVOLSPORT mx \\
\hline & \\
RMSEA & 0.057 \\
GFI & 0.066 \\
AGFI & 0.632 \\
CFI & 0.982 \\
NNFI & 0.981 \\
\hline
\end{tabular}

\section{DISCUSION}

La investigación sobre el voluntariado se ha centrado en aspectos tales como el reclutamiento y selección de los voluntarios (Ringuet-Riot et al, 2014); en las motivaciones intrínsecas (Lee et al, 2014); en los aspectos grupales y comunitarios (Kristiansen et al, 2015); en el costo y beneficio de participar en este tipo de actividades y la satisfacción de las personas que ejercen este rol, realizándose estudios que reconocen factores fundamentales para el mantenimiento del rol de voluntario. Por lo que coincidimos en la importancia de evaluar estos aspectos en el ámbito mexicano.

Por otra parte en los estudios que han tratado el voluntariado en contextos deportivos no son muy amplios, existen pocas investigaciones al respecto; sin embargo consideran la satisfacción como uno de los puntos más acentuados (Fairley et al, 2007; García González et al, 2011; Kim, 2013). Por lo que se presenta la necesidad de contar con herramientas adecuadas para la evaluación de los programas de voluntariado deportivo universitario. La estructura de los factores del cuestionario para la evaluación de los programas de voluntariado deportivo universitario (QVOLSPORT $\mathrm{mx}$ ) tiene relación con otras herramientas como el cuestionario de evaluación de la calidad percibida del voluntariado deportivo (ECPVE) y el (QVOLSPORT) de García-González, ChicaMerino, Hernández-Mendo y Morales-Sánchez, (2011).

\section{APLICACIÓN PRÁCTICA}

El instrumento QVOLSPORT mx utilizado en esta investigación, es válido y fiable para evaluar la calidad percibida de los programas de voluntariado deportivo universitario. Con este instrumento se pueden apoyar para mejorar los programas de voluntariado deportivo mexicano, no solo del ámbito universitario, si no, cualquier evento deportivo nacional, como la Olimpiada Nacional Juvenil, Juegos Nacionales Escolares, entre otros; así como eventos deportivos internacionales en los cuales México sea la sede.

\section{CONCLUSIONES}

Como conclusión, en México existen escasos estudios relacionados con el voluntariado deportivo y menor aún en el ámbito universitario, por lo que consideramos importante continuar investigando esta área. De acuerdo a los resultados obtenidos se concluye que el cuestionario QVOLSPORT mx, posee propiedades psicométricas satisfactorias, tanto de fiabilidad estimada a través de la consistencia interna. En relación a los resultados de la estructura factorial del modelo referentes a los índices de error RMSEA y los índices de ajuste CFI y NNFI presentan un ajuste adecuado; en lo que respecta a la razón de chi cuadrado/ grados de libertad (gl) resulta satisfactoria, utilizando el método de máxima verosimilitud (ML).

El cuestionario QVOLSPORT mx, ha mostrado que atiende a las necesidades de la comunidad universitaria con respecto al voluntariado, posibilitando una mejora continua en los programas de voluntariado deportivo universitario. 


\section{Morquecho et al.}

Se confirma que el instrumento es válido para evaluar la calidad percibida en los programas del voluntariado al contexto deportivo mexicano.

Como futuras líneas de investigación se propone mejorar la evaluación de la calidad de los programas de voluntariado deportivo y lo que significa participar como voluntario en este tipo de eventos, por medio de la utilización de una metodología cualitativa y/o mixed methods; realizando entrevistas a profundidad y/o grupos focales a los voluntarios deportivos.

\section{REFERENCIAS}

1. Anguera, M.T. y Hernández Mendo, A. (2003). Evaluación de programas de actividad física. En A. Hernández Mendo, Psicología del Deporte (Vol.II1): Fundamentos (pp. 141-177). Buenos Aires: Tulio Guterman (http://www.efdeportes.com).

2. Ato, M., López-García, J. J., y Benavente, A. (2013). Un sistema de clasificación de los diseños de investigación en psicología. Anales de Psicología, 29(3), 1038-1059.

3. Brotóns, J. M. y Molina, J. (2011). Manual de gestión del voluntariado en las organizaciones deportivas de la Comunitat Valenciana. Valencia, España: Aula Deportiva Técnica.

4. Chica Merino, E. (2009). Construcción de una herramienta para evaluar la calidad de los programas de voluntariado ambiental. [Tesis Doctoral, Universidad de Málaga].

5. Cronbach, L. J. (1951). Coefficient alpha and the internal structure of tests. Psychometrika, 16, 297 334.

6. Fairley, S., Kellett, P., y Green, B. C. (2007). Volunteering abroad: Motives for travel to volunteer at the Athens Olympic Games. Journal of Sport Management, 21(1), 41-57. doi:10.1123/jsm.21.1.41

7. Gallardo, L., Sánchez-Sánchez, J., Calabuig, F., Ubago-Guisado, E., Burillo, P., Fernández-Luna, Á., y Felipe, J. L. (2016). Herramienta de evaluación de los programas deportivos para la promoción de la salud. Revista de Psicología del Deporte, 25(2), 289-296.
8. García-Pérez, O. (2013). Evaluación de un programa de voluntariado a través de la satisfacción de sus usuarios: los voluntarios. Aula Abierta, 41(3) ,101-12.

9. García-González, R. (2013). Evaluación de la calidad percibida en el voluntariado deportivo [Tesis Doctoral, Universidad de Málaga].

10. García-González, R., Chica-Merino, E., Hernández-Mendo, A., y Morales-Sánchez, V. (2011). Evaluación de la calidad percibida en programas de voluntariado deportivo: un estudio piloto. Cuadernos de Psicología del Deporte, 11(2), 163-170.

11. García-González, R., Morales-Sánchez, V., Hernández-Mendo, A., y Chica-Merino, E. (2011). Una herramienta para evaluar la calidad y la permanencia en el voluntariado deportivo. Cuadernos de Psicología del Deporte, 11(2), 171178.

12. Hemmerle, W., y Hartley, H. (1973). Computing maximum likelihood estimates for the mixed AOV Model using the w-transformation. Technometrics, 15(4), 819-831.

13. Hevia, Felipe J., y Vergara-Lope, S. (2019). Características, satisfacción vital y valores entre voluntarios en México. Evidencias de un proyecto educativo. Espiral, 26(76), 135-182. https://doi.org/10.32870/eees.v26i76.7093

14. International Iniversity Sports Federation FISU (2015). International FISU volunteer programme. Recuperado de: http://www.fisu.net/news/nusfnews/international-fisu-volunteer-programme

15. Kim, M. (2013). Why women volunteer in Korea: roles of identification and satisfaction. Asian Women, 29(1), 79-104.

16. Kristiansen, E; Skirstad, B; Parent, M.M; y Waddington I. (2015). 'We can do it': community, resistance, social solidarity, and long-term volunteering at a sport event. Sport Manage Review, 18(2), 256-67.

17. Lee, C; Reisinger, Y; Kim, M.J; y Yoon S. (2014). The influence of volunteer motivation on satisfaction, attitudes, and support for a megaevent. International Journal of Hospital Management, 40,37-48. 


\section{Calidad en programas de voluntariado de la Universiada mexicana}

18. Littman-Ovadia, H; y Steger, M. (2010). Character strengths and well-being among volunteers and employees: toward an integrative model. The Journal of Positive Psychology, 5(6), 419-30.

19. Miranda, N. S., y Mayne-Nicholls, A. M. (2009). Voluntariado y Edades: Observaciones desde la juventud, adultez y vejez voluntaria de la ciudad de Santiago. Revista del Magíster en Análisis Sistémico Aplicado a la Sociedad, 20, 43-70.

20. Morales-Sánchez, V., Pérez-López, R., Reigal, R. E. y Hernández-Mendo, A. (2020). Mixed Method analysis of emotional quality in sport organizations: The facial expressions in children users as data. Front. Psychol. In press

21. Morgan, H. (2013). Sport volunteering, active citizenship and social capital enhancement: what role in the 'Big Society'. International Journal of Sport Policy and Politics, 5(3), 381-395. https://doi.org/10.1080/19406940.2013.764542

22. Nichols,G., Taylor, P., Barrett, D. y Jeanes, R. (2014). Youth sport volunteers in England: A paradox between reducing the state and promoting a Big Society. Sport Management Review, 17, 337346.

23. Ringuet-Riot, C; Cuskelly, G; Auld, C; y Zakus, D.H. (2014). Volunteer roles, involvement and commitment in voluntary sport organizations: evidence of core and peripheral volunteers. Sport in Society, 17(1):116-33.
24. Ríos, R. (2004). Universitarios y voluntariado: análisis del involucramiento en acciones filantrópicas de los alumnos de la Pontificia Universidad Católica (PUC). Psykhe, 13(2),99115.

25. Searle, S., Casella, G., y McCulloch, C. (1992). Variance components. New York: John Wiley y Sons.

26. Soto-Lagos, R., Constela, C. V., y Vergara, O. F. (2017). Voluntariado y deporte: análisis de factores en la incidencia de la satisfacción de los/as voluntarios/as de los Juegos Suramericanos Santiago de Chile 2014. Revista Brasileira de Ciências do Esporte, 39(2), 191-198.

27. Taylor, P., Panagouleas, T; y Nichols, G. (2012). Determinants of sports volunteering and sports volunteer time in England. International Journal of Sport Policy and Politics, 4(2), 201-220. https://doi.org/10.1080/19406940.2012.656679

28. Vecina, M; Chacón, F; Sueiro, M. (2009). Satisfacción en el voluntariado: estructura interna y relación con la permanencia en las organizaciones. Psicothema, 21(1):112-7.

29. Visauta, B. (1998). Análisis Estadístico con SPSS para WINDOWS (Vol II. Análisis Multivariante). Madrid: Mc-Graw Hill.

30. Visauta, B., Martori, I; y Cañas, J.C. (2005). Análisis estadístico con SPSS para Windows. México: McGraw-Hill. 